\title{
Prognostic value of the mRNA expression of gap junction $\alpha$ members in patients with gastric cancer
}

\author{
XUAN ZHAO, CHAORAN YU, MINHUA ZHENG and JING SUN \\ Department of General Surgery, Shanghai Minimally Invasive Surgery Center, Ruijin Hospital, \\ Shanghai Jiao Tong University School of Medicine, Shanghai 200025, P.R. China
}

Received July 24, 2018; Accepted May 2, 2019

DOI: $10.3892 / \mathrm{ol} .2019 .10516$

\begin{abstract}
Gastric cancer remains one of the primary causes of cancer-associated death worldwide. The gap junction $\alpha$ (GJA) family has been demonstrated to be involved in the cellular proliferation and metastasis of gastric cancer. However, the prognostic value of GJA in gastric cancer is yet to be elucidated. In the present study, the overall survival (OS) of patients with gastric cancer and the mRNA expression of GJA family members, including GJA1, GJA3, GJA4, GJA10 and GJA12, were analyzed using 593 patients with gastric cancer from the Kaplan-Meier plotter database. High GJA1 and GJA10 mRNA expression levels were associated with a poorer patient outcome $(\mathrm{P}=0.0066$ and $\mathrm{P}=0.015$, respectively), whereas high mRNA expression levels of GJA4 and GJA12 were associated with longer survival times $(\mathrm{P}=0.0056$ and $\mathrm{P}=0.0054$, respectively). Furthermore, the values of specific prognostic indicators of different subtypes of gastric cancer, including human epidermal growth factor receptor 2 status, Lauren differentiation and tumor stage, were also analyzed. The findings of the present study suggested a potential role for GJA family members in gastric cancer, which warrants further investigation.
\end{abstract}

\section{Introduction}

Gastric cancer is one of the most common causes of cancer-associated mortality (1). Despite significant improvements in therapy, including neoadjuvant chemoradiotherapy, advanced surgical techniques and novel target drugs, gastric cancer has a high tendency for recurrence (2). Furthermore, the progression and genetic characteristics of gastric cancer

Correspondence to: Professor Minhua Zheng or Professor Jing Sun, Department of General Surgery, Shanghai Minimally Invasive Surgery Center, Ruijin Hospital, Shanghai Jiao Tong University School of Medicine, 197 Ruijin Er Road, Shanghai 200025, P.R. China

E-mail: zmhtiger@yeah.net

E-mail: sj11788@rjh.com.cn

Key words: gap junction, gastric cancer, connexin, mRNA expression, prognosis remain unclear. In recent decades, it has been revealed that gastric cancer results from multi-gene alterations in a number of signaling pathways. A deeper understanding of gastric cancer-associated genes may improve the diagnosis and treatment of the disease.

The gap junction $\alpha$ (GJA) genes encode connexin $(\mathrm{Cx})$ proteins. Cxs compose cell gap junctions, facilitating intercellular communication to regulate a number of cell functions, including cell death, proliferation and differentiation. To date, $12 \mathrm{Cx}$ proteins, GJA1-GJA12, have been identified. These proteins are expressed in different tissues to varying degrees $(3,4)$, and the genetic and biological features of each $\mathrm{Cx}$ can differ. These proteins have been demonstrated to be involved in cancer development, metastasis and the regulation of cancer cell survival during radiotherapy (5-9). Several studies have indicated that the GJA family serve an important role in gastric cancer, particularly in Helicobacter pylori infection-associated and intestinal-type gastric cancer $(5,6)$. However, these studies focused primarily on in vitro investigations of the molecular mechanisms of these genes in gastric cancer; their roles in clinical practice are largely unknown.

In the present study, the prognostic roles of GJA mRNA expression levels were assessed in patients with gastric cancer using the Kaplan-Meier plotter (KM plotter, http://kmplot. com/analysis/index.php? $\mathrm{p}=$ service \&cancer $=$ gastric) and Gene expression profiling interactive analysis (GEPIA, http://gepia. cancer-pku.cn) platforms. The Kaplan-Meier plotter is an open database within the Gene Expression Omnibus (www.ncbi. nlm.nih.gov/geo/) that collects gene expression and survival data from patients with gastric, lung and breast cancer. GEPIA is an online tool for extracting RNA sequencing data from The Cancer Genome Atlas (TCGA) and Genotype-Tissue Expression (GTEx) databases. The use of these online tools revealed a significant association between GJA mRNA expression levels and the survival of patients with gastric cancer.

\section{Materials and methods}

The association between GJA mRNA expression level and patient overall survival (OS) was determined using the Kaplan-Meier plotter (http://kmplot.com/analysis/index.php? $\mathrm{p}=$ service \&cancer $=$ gastric) $(10)$, which includes the data of 876 patients with gastric cancer. A total of 593 patients from the GSE14210, GSE15459, GSE22377, GSE29272 and GSE51105 


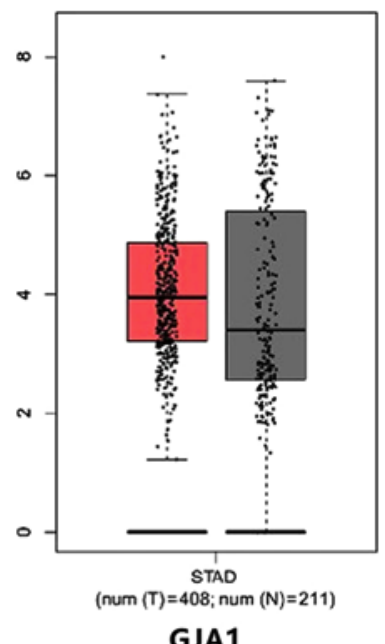

GJA1

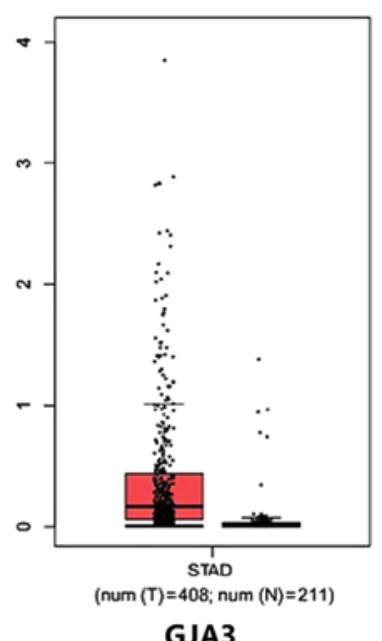

GJA3
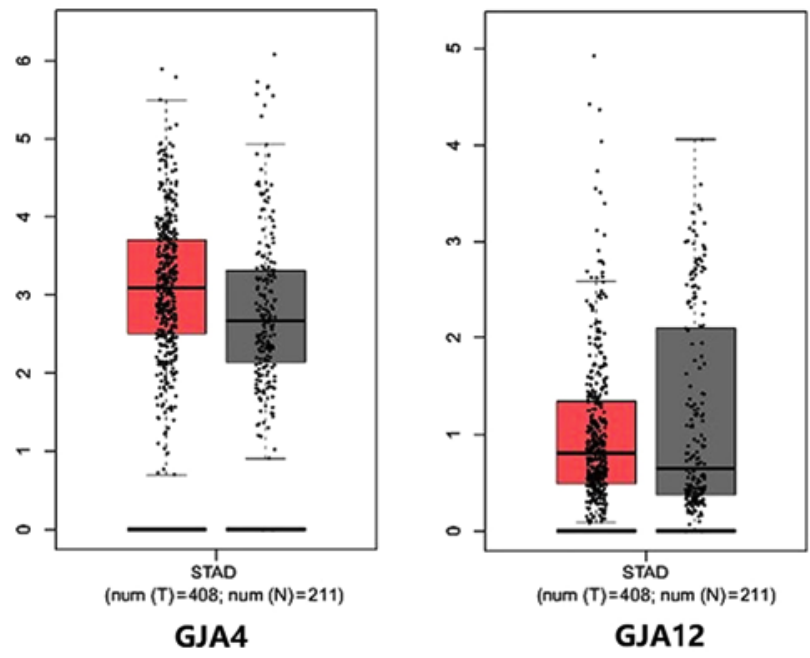

Figure 1. Boxplots of the mRNA expression levels of GJA1, GJA3, GJA4 and GJA12 in gastric cancer and normal patient tissues. The red and grey boxes represent cancerous and normal tissues respectively. The y-axis represents $\log 2(\mathrm{TPM}+1)$ transformed RNA seq expression data. GJA3 and GJA4 mRNA expression levels were elevated in tumor tissues but no significant differences were observed. GJA, gap junction $\alpha$; STAD, stomach adenocarcinoma.

datasets were assessed; the GSE62254 database, which exhibited markedly different patient characteristics to the other datasets, was excluded. Using the Kaplan-Meier plotter, the data associated with different clinicopathological variables, including sex, tumor stage, Lauren classification, tumor differentiation and human epidermal growth factor receptor 2 (Her-2) status were collected. Data regarding GJA gene family members, including GJA1, GJA3, GJA4, GJA10 and GJA12 was also assessed, and the mRNA expression levels of these members were analyzed. $\mathrm{P}<0.05$ was considered to indicate a statistically significant difference. All possible cutoff values between the lower and upper quartiles were determined, and the best performing threshold used as the cutoff.

The mRNA expression levels of tumor and normal tissues were analyzed using the GEPIA database (http://gepia. cancer-pku.cn), which contains the data of 9,736 tumors and 8,587 normal samples from TCGA and GTEx datasets. The expression levels of GJA1, GJA3, GJA4 and GJA12 in 619 patients with gastric cancer were assessed using GEPIA. The expression levels in tumor and normal tissues at different disease stages were displayed as boxplots and stage plots, for which the method of differential analysis was one-way ANOVA. For the boxplots, disease state (tumor or normal) was used as the variable for calculating differential expression; pathological stage was used for the stage plots. $\mathrm{P}<0.05$ was considered to indicate a statistically significant difference.

\section{Results}

Differential expression of GJA members between gastric cancer and normal tissues. In the present study, the prognostic value of GJAs in gastric cancer was assessed. The GEPIA was used to determine the mRNA expression levels of GJA1, GJA3, GJA4, GJA10 and GJA12 in patients with gastric cancer. The median expression levels of GJA1, GJA3, GJA4 and GJA12 were determined (Fig. 1). However, the expression data for GJA10 was not collected from the GEPIA database. Notably, GJA3 and GJA4 exhibited a higher expression level in gastric cancer tissues (Fig. 1). Furthermore, the mRNA expression levels of GJAs at different tumor stages were assessed and are indicated as stage plots (Fig. 2). Notably, there was a statistically significant association between GJA4 expression and the different pathological stages (Fig. 2C). No significant association was revealed for the other family members (Fig. 2A, B and D). These findings indicated that specific GJA members were differentially expressed in gastric cancer tissues compared with normal tissues.

Prognostic value of GJA members in gastric cancer. The Kaplan-Meier plotter was used to assess the prognostic value of GJA members in gastric cancer; patient characteristics are listed in Table I. The data revealed that the mRNA expression levels of GJA1, GJA4, GJA10 and GJA12 were significantly associated with OS in patients with gastric cancer (Fig. 3). High mRNA expression levels of GJA1 and GJA10 were associated with poorer OS (Fig. 1A and C), whereas high mRNA expression levels of GJA4 and GJA12 were associated with improved OS (Fig. 1B and D). Regarding GJA3, no significant difference was indicated between mRNA expression level and OS $(\mathrm{HR}=1.27 ; 95 \% \mathrm{CI}=0.92-1.75 ; \mathrm{P}=0.14)$. In conclusion, GJA1, GJA4, GJA10 and GJA12 were associated with the survival outcome of patients with gastric cancer.

Prognostic value of GJA members in patients with different pathological characteristics. To further understand the association between the mRNA expression levels of GJAs and survival outcome, the OS of patients with different pathological characteristics was evaluated, including Her-2 status, Lauren classification, tumor differentiation status and tumor-node-metastasis (TNM) stage. As indicated in Fig. 4, the prognostic value of GJAs with Her-2 status was determined. A high GJA1 and GJA10 mRNA expression level was associated with poorer OS in Her-2-positive patients (Fig. 4A and C), whilst low GJA4 and GJA12 expression levels were associated with reduced survival time in Her-2-negative patients (Fig. 4E and F).

The Lauren classification is used to define gastric cancer into intestinal, diffuse and mixed subtypes. The prognostic 

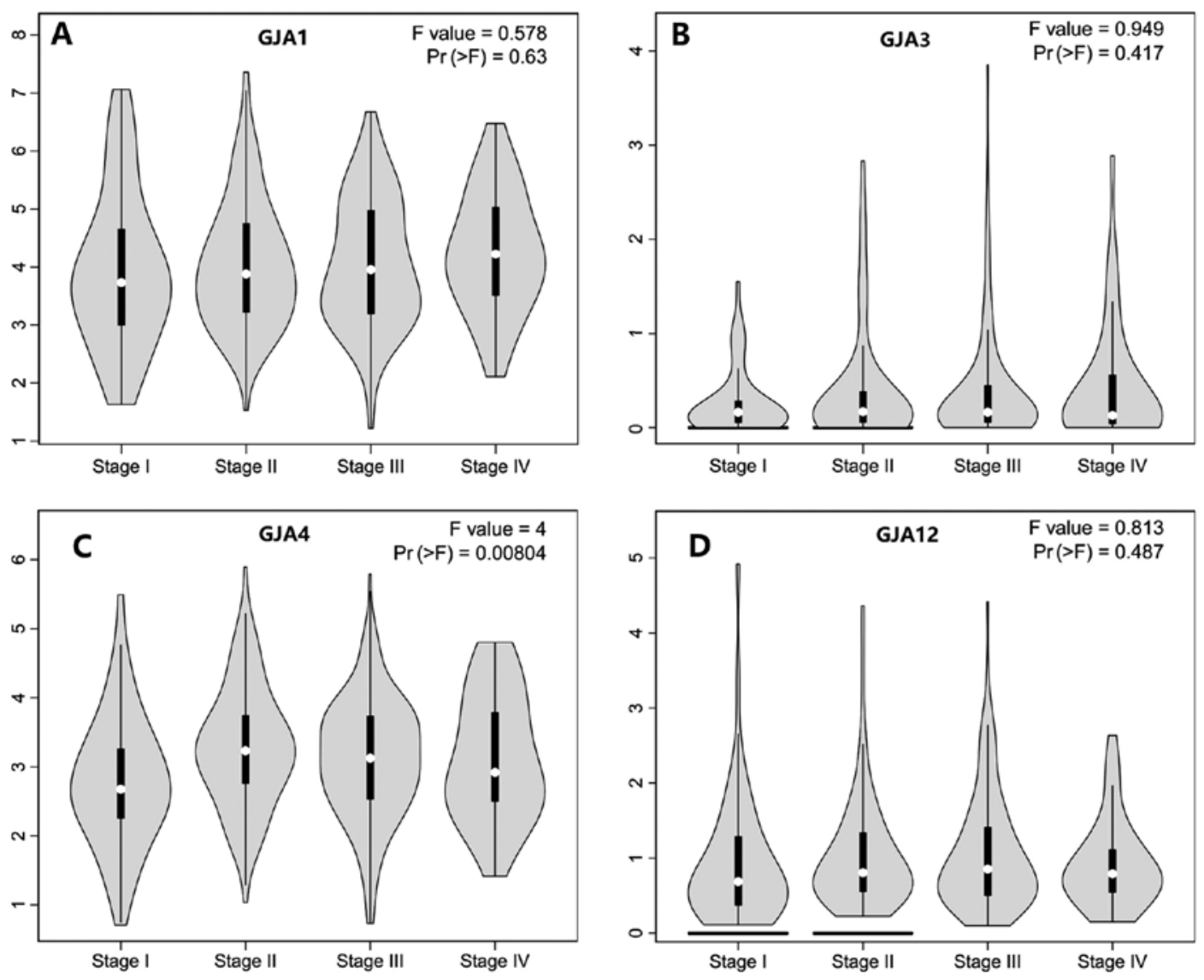

Figure 2. Expression levels of GJA members at different cancer stages. The y-axis represents $\log 2(\mathrm{TPM}+1)$ transformed RNA seq expression data. (A) GJA1, (B) GJA3, (C) GJA4 and (D) GJA12. GJA, gap junction $\alpha$.

value of GJA members in different Lauren classifications was revealed (Fig. 5). A high GJA1 mRNA expression level was associated with poorer OS in patients with intestinal and diffuse type gastric cancer (Fig. 5A and B). An elevated GJA3 mRNA expression level was associated with poorer OS in patients with intestinal type gastric cancer (Fig. 5C). Furthermore, high GJA10 mRNA expression level was also associated with poorer OS time in patients with intestinal or diffuse type gastric cancer (Fig. 5D and E).

Regarding tumor differentiation, GJA mRNA expression level also demonstrated prognostic value. As indicated in Fig. 6, High GJA1 mRNA expression was associated with poorer OS in patients with well- or moderately-differentiated gastric cancer (Fig. 6A and B). High GJA10 expression level was associated with poorer OS times in patients with moderately or poorly differentiated gastric cancer (Fig. 6C and D). GJA12 expression was significantly associated with the OS in patients with moderately differentiated tumors (Fig. 6E). All other conditions not indicated in the figure. were analyzed, but had no statistical significance in terms of prognostic value.

For different gastric cancer TNM stages, the GJA mRNA expression level was of prognostic value on a number of levels. As indicated in Table II, some of the GJA members were associated with prognosis in different stages of gastric cancer. Among those listed, GJA1, GJA10 and GJA12 demonstrated the most significant association with gastric cancer survival outcome. Notably, the number of stage T1 and T4 patients was too small to be valid for analysis in the KM plotter database.

\section{Discussion}

The GJA gene family encodes Cxs, which compose cell hemi-channels, connexons and gap junction channels (11-13). It has been reported that these gap junction structures are involved in several cellular responses under physiological and pathological states (14). GJA-encoded Cxs are widely distributed in the human body and are abundantly expressed in gastrointestinal cells. Among the GJA family members, GJA1 (Cx43) and GJA4 (Cx37) have been confirmed to be expressed in normal gastrointestinal cells (15). However, Cx isotypes not identified in normal tissue may be detected in associated cancers (16). In gastric cancer cells, GJA has been demonstrated to be associated with intestinal type gastric cancer (17) and Helicobacter pylori-associated gastric tumorigenesis $(18,19)$; however, the functional mechanism remains unclear. According to a recent study on the GJA family members, the majority of studies focus on the molecular mechanisms and pathological progression of GJA. However, the number of studies on the clinical value of GJA was limited.

Among the GJA members investigated in the present study, GJA1 was the most widely reported. GJA1-encoded Cx43 is the most abundant cx in gastric epithelial and intestinal epithelial cells (15). Cx43 is a component of cell gap junctions that serves an important role in intercellular communication, cell-cell channel formation and the exchange of signaling molecules (20). Several hypotheses and potential mechanisms of $\mathrm{Cx} 43$-associated gastric cancer have been reported. Cx43 
Table I. Characteristics of patients with gastric cancer in the Kaplan-Meier plotter database.

\begin{tabular}{|c|c|c|}
\hline Characteristics & Patients, n & Ratio \\
\hline \multicolumn{3}{|l|}{ Age } \\
\hline$<65$ & 83 & 0.43 \\
\hline$\geq 65$ & 109 & 0.57 \\
\hline \multicolumn{3}{|l|}{ Sex } \\
\hline Male & 567 & 0.69 \\
\hline Female & 244 & 0.31 \\
\hline \multicolumn{3}{|l|}{ Stage } \\
\hline $\mathrm{I}$ & 69 & 0.10 \\
\hline II & 145 & 0.21 \\
\hline III & 319 & 0.47 \\
\hline IV & 152 & 0.22 \\
\hline \multicolumn{3}{|l|}{ T Stage } \\
\hline 1 & 14 & 0.03 \\
\hline 2 & 253 & 0.49 \\
\hline 3 & 208 & 0.40 \\
\hline 4 & 39 & 0.08 \\
\hline \multicolumn{3}{|l|}{ N Stage } \\
\hline 0 & 76 & 0.15 \\
\hline 1 & 232 & 0.45 \\
\hline 2 & 129 & 0.25 \\
\hline 3 & 76 & 0.15 \\
\hline \multicolumn{3}{|l|}{ M Stage } \\
\hline 0 & 459 & 0.74 \\
\hline 1 & 58 & 0.26 \\
\hline \multicolumn{3}{|l|}{ Her- 2 status } \\
\hline Positive & 425 & 0.40 \\
\hline Negative & 641 & 0.60 \\
\hline \multicolumn{3}{|c|}{ Lauren classification } \\
\hline Intestinal & 336 & 0.54 \\
\hline Diffuse & 248 & 0.40 \\
\hline Mixed & 33 & 0.06 \\
\hline \multicolumn{3}{|l|}{ Differentiation } \\
\hline Poor & 166 & 0.63 \\
\hline Moderate & 67 & 0.25 \\
\hline Well & 32 & 0.12 \\
\hline
\end{tabular}

T, tumor; N, node; M, metastasis; Her-2, human epidermal growth factor receptor 2. The present study included 5datasets: GSE14210, GSE15459, GSE22377, GSE29272, GSE51105. Not all of the clinical characteristics were reported by every dataset.

may induce Helicobacter pylori-associated gastric cancer via VacA-induced cell death $(20,21)$. Additionally, Tang et al $(22,23)$ reported that the abnormal expression of $\mathrm{Cx} 43$ may promote lymph node metastasis and peritoneal metastasis of gastric cancer through $\mathrm{Cx} 43$-mediated heterocellular gap-junctional intercellular communication. Furthermore, Liu et al (24) suggested that $\mathrm{Cx} 43$ may increase the chemotherapeutic sensitivity of gastric cancer cells. These in vitro studies suggest an
Table II. Association between mRNA expression levels of GJA members and the overall survival of patients with gastric cancer at different Tumor-Node-Metastasis stages.

\begin{tabular}{|c|c|c|c|c|}
\hline Variable & mRNA & HR & $95 \% \mathrm{CI}$ & $\begin{array}{c}\text { Log rank } \\
\text { P-value }\end{array}$ \\
\hline \multirow[t]{5}{*}{$\mathrm{T} 2(\mathrm{n}=66)$} & GJA1 & 2.34 & $1.04-5.27$ & $0.04^{\mathrm{a}}$ \\
\hline & GJA3 & 1.85 & $0.9-3.8$ & $0.09^{\mathrm{a}}$ \\
\hline & GJA4 & 1.71 & $0.82-3.56$ & 0.15 \\
\hline & GJA10 & 2.5 & $1.2-5.22$ & $0.01^{\mathrm{a}}$ \\
\hline & GJA12 & 0.41 & $0.19-0.86$ & $0.02^{\mathrm{a}}$ \\
\hline \multirow[t]{5}{*}{ T3 (n=117) } & GJA1 & 1.43 & $0.93-2.2$ & 0.11 \\
\hline & GJA3 & 0.69 & $0.44-1.1$ & 0.12 \\
\hline & GJA4 & 0.66 & $0.43-1.03$ & 0.06 \\
\hline & GJA10 & 1.71 & $1.09-2.7$ & $0.02^{\mathrm{a}}$ \\
\hline & GJA12 & 0.43 & $0.27-0.7$ & $<0.01^{\mathrm{b}}$ \\
\hline \multirow[t]{5}{*}{ N0 $(n=38)$} & GJA1 & 1.56 & $0.44-5.48$ & 0.49 \\
\hline & GJA3 & 0.53 & $0.19-1.49$ & 0.22 \\
\hline & GJA4 & 2.89 & $0.65-12.77$ & 0.14 \\
\hline & GJA10 & 4.3 & $1.58-11.75$ & $<0.01^{\mathrm{b}}$ \\
\hline & GJA12 & 0.5 & $0.18-1.41$ & 0.18 \\
\hline \multirow[t]{5}{*}{$\mathrm{N} 1+2+3(\mathrm{n}=175)$} & GJA1 & 2.16 & $1.43-3.27$ & $<0.01^{\mathrm{b}}$ \\
\hline & GJA3 & 1.38 & $0.92-2.07$ & 0.11 \\
\hline & GJA4 & 0.79 & $0.52-1.2$ & 0.27 \\
\hline & GJA10 & 1.25 & $0.85-1.84$ & 0.25 \\
\hline & GJA12 & 0.61 & $0.41-0.92$ & $0.02^{\mathrm{a}}$ \\
\hline \multirow[t]{5}{*}{ M0 (n=186) } & GJA1 & 1.93 & $1.27-2.92$ & $<0.01^{\mathrm{b}}$ \\
\hline & GJA3 & 1.29 & $0.87-1.9$ & 0.2 \\
\hline & GJA4 & 0.68 & 0.43-1.09 & 0.11 \\
\hline & GJA10 & 1.49 & $1-2.22$ & 0.05 \\
\hline & GJA12 & 0.47 & $0.31-0.72$ & $<0.01^{\mathrm{b}}$ \\
\hline \multirow[t]{5}{*}{ M1 (n=31) } & GJA1 & 1.4 & $0.63-3.11$ & 0.4 \\
\hline & GJA3 & 0.58 & $0.26-1.29$ & 0.18 \\
\hline & GJA4 & 1.61 & $0.73-3.54$ & 0.23 \\
\hline & GJA10 & 0.47 & $0.19-1.16$ & 0.09 \\
\hline & GJA12 & 1.97 & $0.73-5.31$ & 0.17 \\
\hline
\end{tabular}

${ }^{\mathrm{a}} \mathrm{P}<0.05,{ }^{\mathrm{b}} \mathrm{P}<0.01$. In the Kaplan-Meier plotter database, the number of patients with $\mathrm{T} 1$ and $\mathrm{T} 4$ stage cancer was too small for valid statistical analyses. GJA, gap junction $\alpha$; HR, hazard ratio; CI, confidence interval.

association between GJA1 and gastric cancer. In the present study, a high GJA1 mRNA expression level was associated with a poorer OS in patients with gastric cancer, particularly in those that were also Her-2-positive. Previous results have indicated that GJA1 enhances tumor proliferation and migration in Her-2-positive patients with breast cancer (25), which suggests a mutual effect between Her-2 and GJA1. In intestinal and diffuse types of gastric cancer, GJA1 was also associated with a poorer patient outcome.

GJA3 is primarily distributed in heart and testicular tissues but is also expressed at low levels in the stomach. It is associated with various types of breast cancer and cataract (26-29), and also has a distinct prognostic value for 
A

201667_at

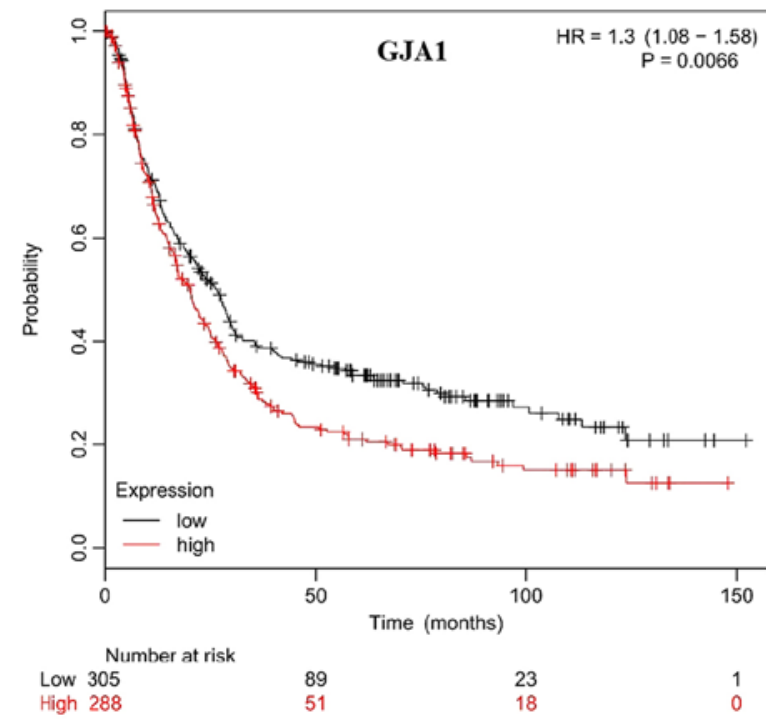

C

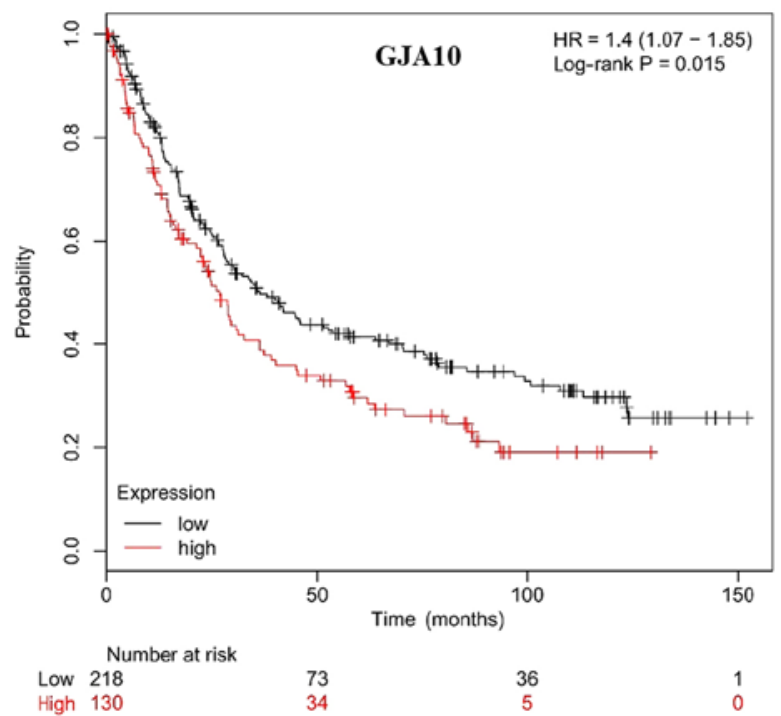

B

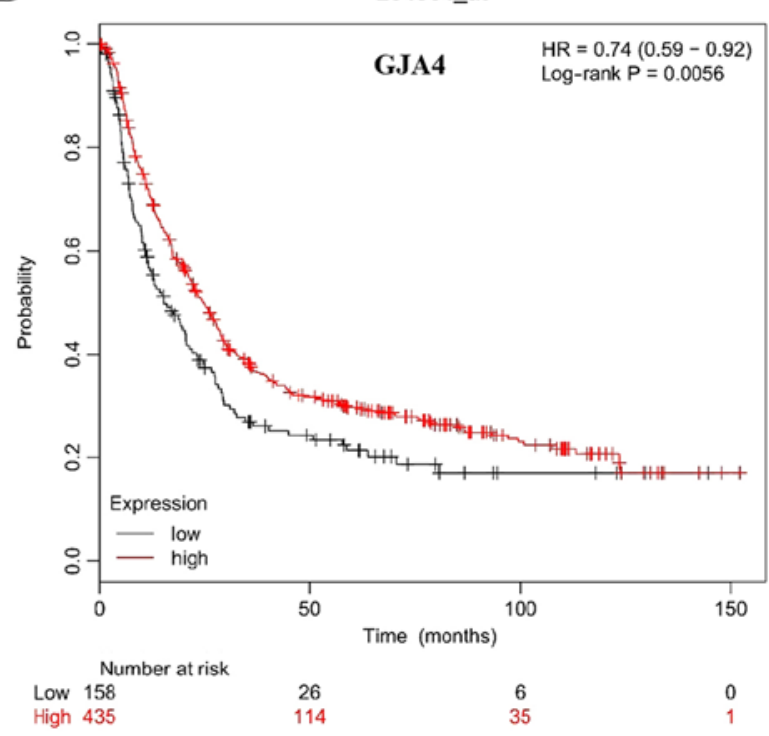

D

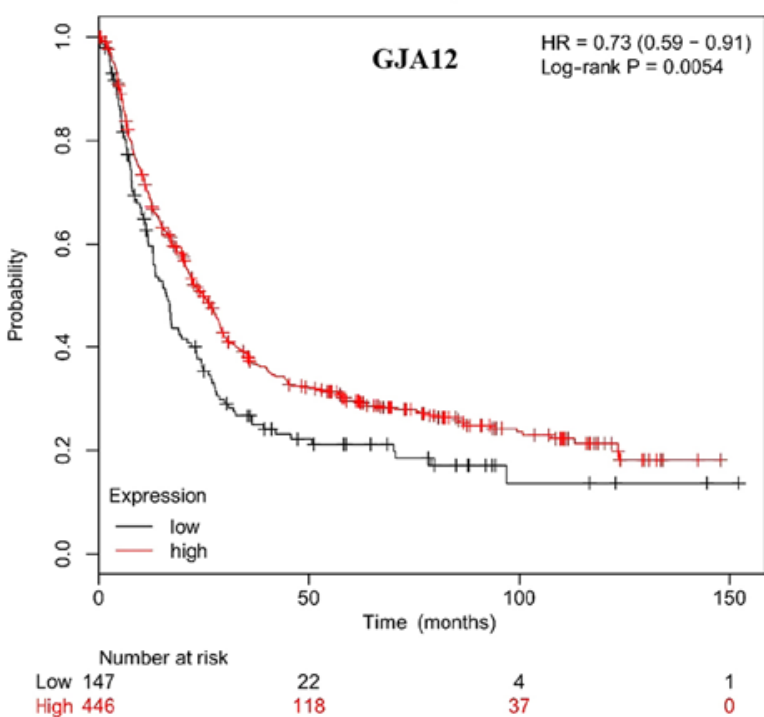

Figure 3. Association between GJA members and the occurrence of gastric cancer. (A) Survival curve of GJA1 (Affymetrix IDs: 20167_at, HR=1.3; 95\% CI, 1.08-1.58; P=0.0066). (B) Survival curve of GJA4 (Affymetrix IDs: 204904_at, HR=0.74; 95\% CI, 0.59-0.92; P=0.0056). (C) Survival curve of GJA10 (Affymetrix IDs: 1553044_at, HR=1.4; 95\% CI, 1.07-1.85; P=0.015). (D) Survival curve of GJA12 (Affymetrix IDs: 207025_at, HR=0.73; 95\% CI, 0.59-0.91; $\mathrm{P}=0.0054)$. GJA, gap junction $\alpha$; HR, hazard ratio; $\mathrm{CI}$, confidence interval.

breast cancer $(26,29)$. However, no scientific study has been published concerning its association with gastric cancer. GJA4 has been reported to participate in tumor proliferation (30) and growth suppression (31); Jing et al (32) reported that GJA4 was correlated with gastric cancer and that small interfering RNA inhibition of GJA4 in gastric tumors in mice could promote tumor cell apoptosis. Notably, there is limited research regarding GJA10. It has been suggested that the GJA10 (Cx62) is mainly distributed in the retina (33) and may be involved in visual perception functions (34). To the best of our knowledge, no study has reported the role of GJA10 in gastric cancer. GJA12 is methylated in the carcinogenesis of Barrett's esophagus, which indicates its potential function in tumor progression (35). GJA12 is also associated with an increased risk of secondary lymphedema following breast cancer treatment (36).
GJA family members predominantly mediate gap-junctional intercellular communication (GJIC), which is the only way for adjacent cells to exchange signals internally (37). Gap junctions also serve an important role in maintaining tissue homeostasis. Deletion of $\mathrm{Cx}$ genes may result in disorders such as cancer and cardiac malformation (38). Several studies have indicated reduced GJIC among neighboring cells in malignant tumor tissues (39-41). Additionally, Ruch et al (40) suggested that reduced GJIC promoted cancer cell growth and tumorigenicity, and Trosko et al (42) demonstrated that one of the differences between a cancer cell and its normal parental cell is GJIC-competence. These results suggest the importance of GJIC in carcinogenesis and regulation of the cell cycle. However, Yamasaki et al (38) indicated that the expression of Cxs, rather than GJIC level, is more closely associated with the control of tumor growth, which suggests a GJIC-independent function for GJA members. 

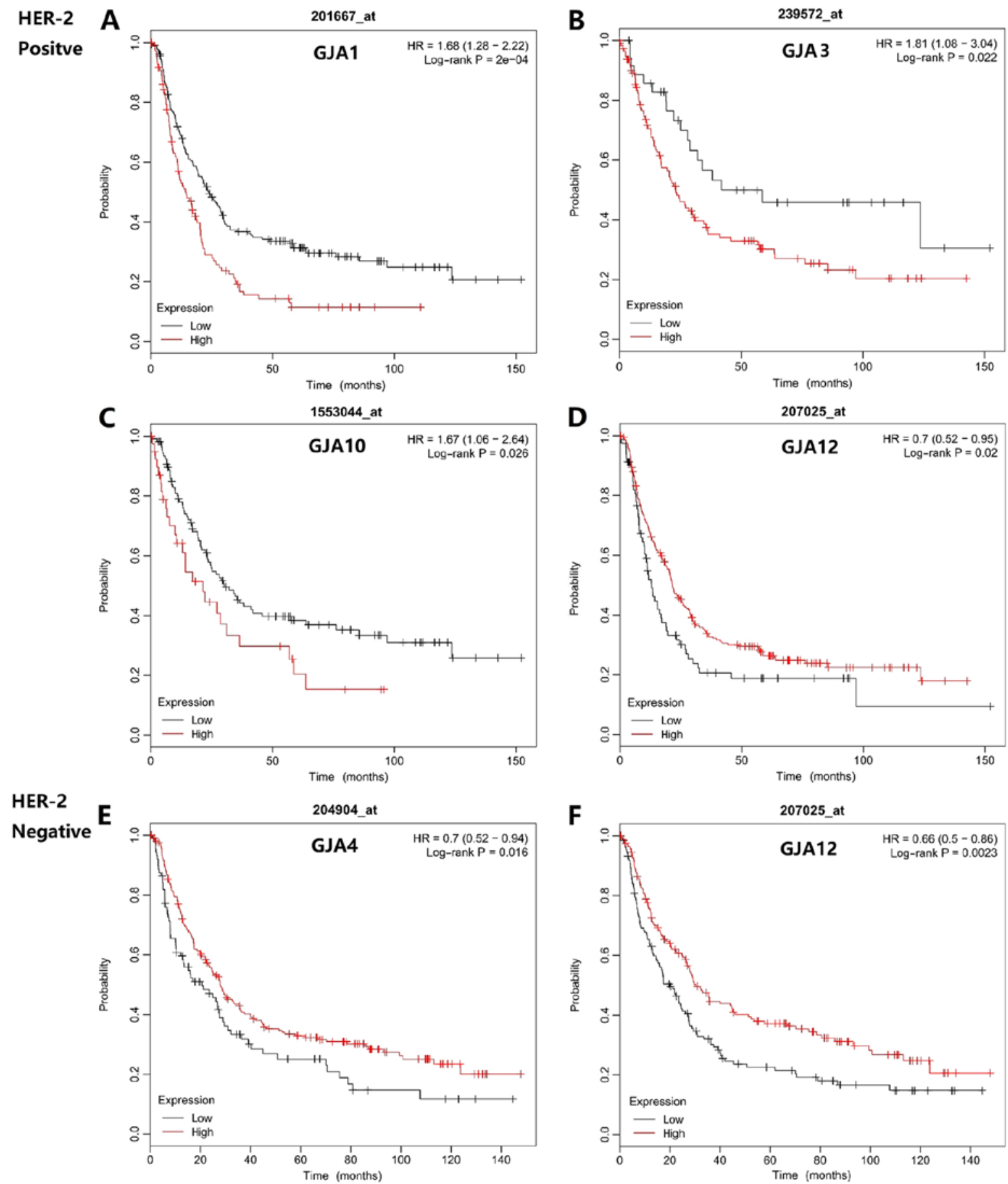

Figure 4. Association between mRNA expression levels of GJA members and the overall survival of Her-2-positive and -negative patients with gastric cancer. For Her-2-positive patients, the prognostic relevance was revealed for (A) GJA1 (HR=1.68; 95\% CI, 1.28-2.22; P=0.0002), (B) GJA3 (Affymetrix IDs: 239572_at, $\mathrm{HR}=1.81 ; 95 \% \mathrm{CI}, 1.08-3.04 ; \mathrm{P}=0.022)$, (C) GJA10 (HR=1.67; 95\% CI, 1.06-2.64; $\mathrm{P}=0.026)$ and (D) GJA12 (HR=0.7; 95\% CI, 0.52-0.95; P=0.02) expression. For (E) GJA4 (HR=0.70; 95\% CI, 0.52-0.94; $\mathrm{P}=0.016$ ) and (F) GJA12 (HR=0.66; 95\% CI, 0.5-0.86; $\mathrm{P}=0.0023$ ) expression, the differences in prognosis were presented in Her-2-negative patients. GJA, gap junction $\alpha$; HR, hazard ratio; CI, confidence interval; Her-2, human epidermal growth factor receptor 2.

Although the relatively well-studied GJA1 is reportedly associated with the carcinogenesis of gastric cancer, in the present study there were no significant differences in the expression levels of GJA1 in tumor tissues compared with normal tissues. Yamasaki et al (39) identified that Cxs were normally expressed, but aberrantly localized in many tumor cells, indicating that the dysregulation of GJIC may be caused by the abnormal expression and localization of Cxs. Therefore, it was speculated that the differential expression of GJA members was not the only mechanism in gap junction-associated carcinogenesis. Tang et al (22) suggested that GJA1-mediated heterocellular GJIC between gastric cancer cells serves an essential role in metastasis. These reports indicated that GJA members were not only involved in cell cycle control of individual malignant cells, but also neighboring cells that may promote tumor proliferation and metastasis.

Among the GJA members, it was revealed that GJA1 (Cx43) was most obviously associated with gastric cancer. 

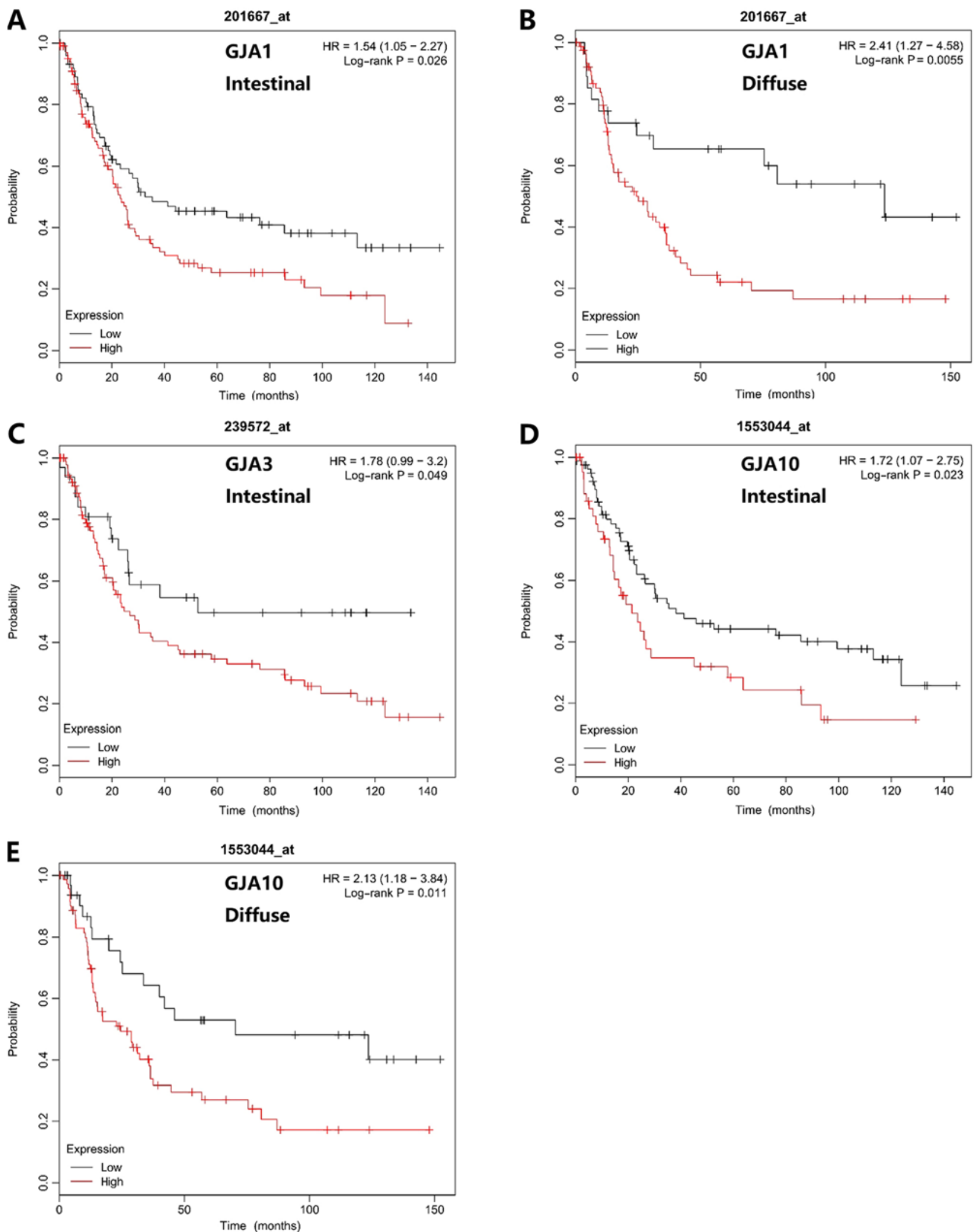

Figure 5. Association between mRNA expression levels of GJA members and the overall survival of patients with different Lauren classifications. (A and B) GJA1 $(\mathrm{HR}=1.54 ; 95 \% \mathrm{CI}, 1.05-2.27$; $\mathrm{P}=0.026$; and $\mathrm{HR}=2.41 ; 95 \% \mathrm{CI}, 1.27-4.58 ; \mathrm{P}=0.0055)$, (C) GJA3 (HR=1.78; 95\% CI, 0.99-3.2; P=0.049) and (D and E) GJA10 $(\mathrm{HR}=1.72 ; 95 \% \mathrm{CI}, 1.07-2.75 ; \mathrm{P}=0.023$, and $\mathrm{HR}=2.13 ; 95 \% \mathrm{CI}, 1.18-3.84 ; \mathrm{P}=0.011)$ mRNA expression levels exhibited prognostic values for different Lauren differentiations. GJA, gap junction $\alpha$; HR, hazard ratio; CI, confidence interval.

Her-2 expression in gastric cancer is associated with a poor outcome (43), and for Her-2-positive patients with gastric cancer, high GJA1, GJA3 and GJA10 mRNA expression levels were predictive of poorer OS times, whereas high GJA12 mRNA expression levels were associated with an improved outcome. Although several studies have aimed to determine 
GJA1 A

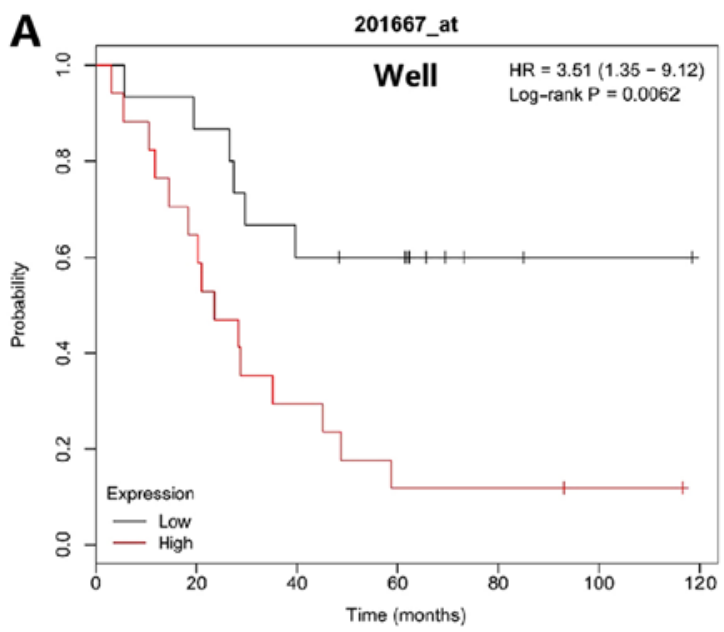

GJA10

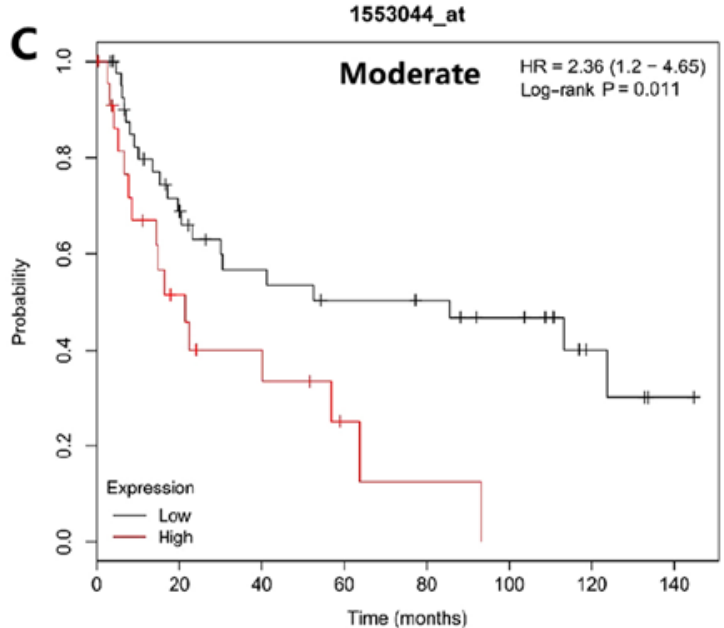

GJA12

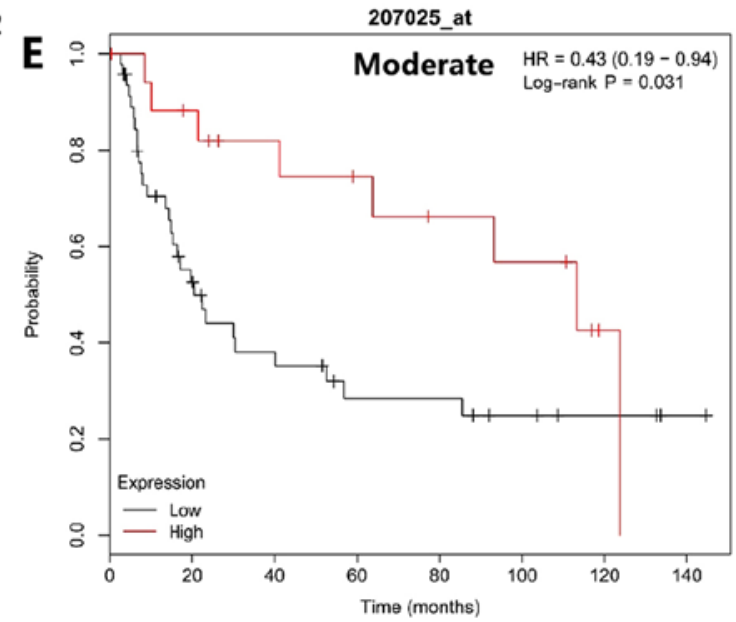

B
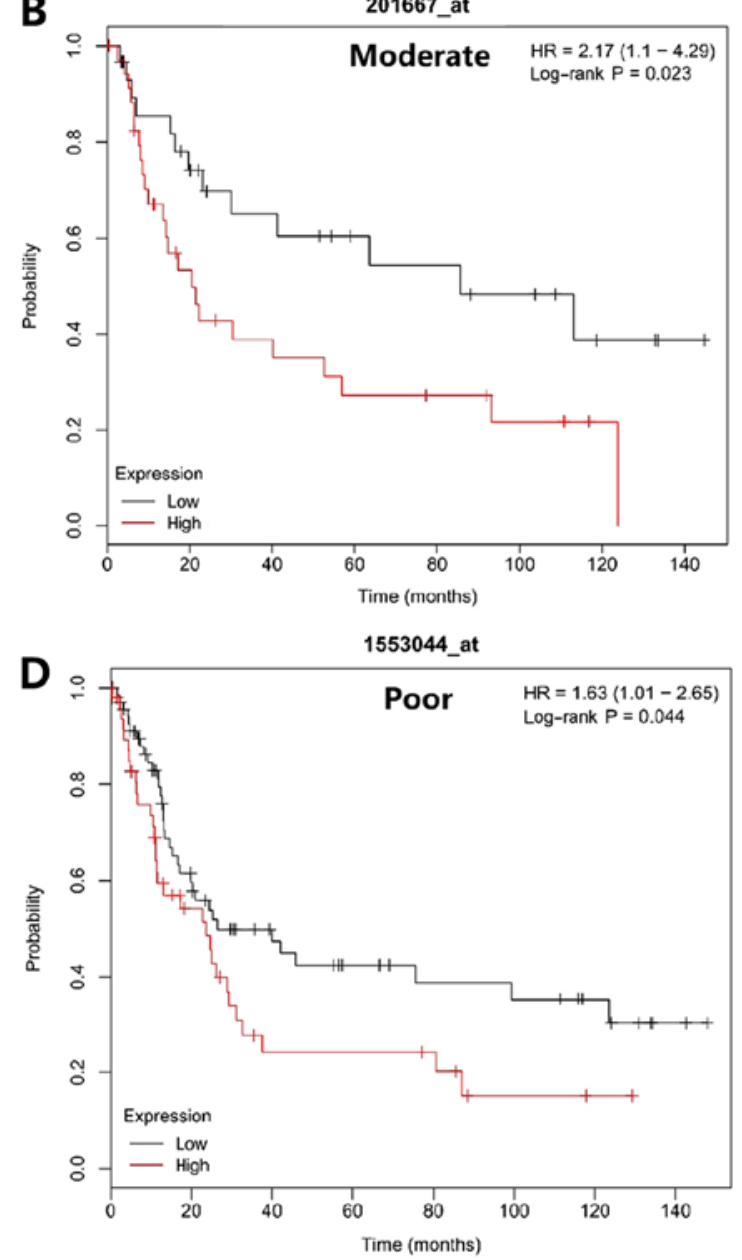

Figure 6. Association between mRNA expression levels of GJA members and the overall survival of patients with different pathological differentiations. Statistically significant differences were revealed regarding (A and B) GJA1 (HR=3.51; 95\% CI, 1.35-9.12; $\mathrm{P}=0.0062$, and HR=2.17; 95\% $\mathrm{CI}, 1.1-4.29 ; \mathrm{P}=0.023)$, (C and D) GJA10 (HR=2.36; 95\% CI, 1.2-4.65; $\mathrm{P}=0.011$, and $\mathrm{HR}=1.63$; 95\% CI, 1.01-2.65; $\mathrm{P}=0.044)$ and (E) GJA12 (HR=0.43; 95\% CI, 0.19-0.94; $\mathrm{P}=0.031)$. GJA, gap junction $\alpha$; HR, hazard ratio; CI, confidence interval.

the link between GJA mRNA expression and Her-2 status in other cancer types $(25,27)$, there is still a lack of evidence to explain the potential mechanism in gastric cancer.

For different tumor stages, the prognostic value of GJA also generated some significant results. It has been indicated that gap junctions act as suppressors in the early stages of tumor development; however, in the advanced stages, they may facilitate tumor progression by promoting the invasion of migrating tumor cells into the surrounding tissues $(5,8,9)$. These 2 functions are considered to be the result of different gap junction mechanisms at different tumor stages (8). In the present study, the expression of GJA at $\mathrm{N}$ and $\mathrm{M}$ stage exhibited a significant difference between $\mathrm{N} 0$ and $\mathrm{N} 1+2+3 / \mathrm{M} 0$ and $\mathrm{M} 1$, which supports previous research. Several studies have also indicated that Cxs may be associated with lymph node metastasis $(22,36,44)$. Kanczuga-Koda et al $(44)$ suggested that the 
expression of GJA1 was increased in metastatic lymph nodes compared with primary breast tumors, and Ezumi et al (45) found that GJA1-negative primary colorectal tumors could metastasis into GJA1-positive tumors in the lymph nodes. The potential role of GJAs in lymph node metastasis may also explain their differential expression at different $\mathrm{N}$ stages.

The present study had a number of limitations. Previous studies have confirmed that the relationship between specific GJA family members and gastric cancer remains unclear. However, in the present study, the potential joint influence of these members on patient survival was not analyzed. Additionally, it is a bioinformatics-based analysis of GEO and TCGA datasets, with a lack of in vitro and in vivo validation experiments.

The present study focused on the abnormal expression of GJAs and their prognostic value in gastric cancer. The findings suggested that GJA family members are associated with the survival outcome of patients with gastric cancer; specifically, high GJA1 and GJA10 expression levels were associated with poorer outcomes, whilst high GJA4 and GJA12 were associated with improved outcomes. Moreover, for different pathological features, including Her-2 status, Lauren classification, tumor differentiation and TNM stage, GJA mRNA expression level also exhibited predictive value. These results indicated the potential of GJA members as gene targets for the prognosis and treatment of gastric cancer, though further insights are required to determine their clinical applications and mechanisms.

\section{Acknowledgements}

Not applicable.

\section{Funding}

The present study was supported by the National Natural Science Foundation of China (grant nos. 81402423, 81572818 and 81871984) and the Shanghai Municipal Commission of Health and Family Planning (grant no. 2017YQ062).

\section{Availability of data and materials}

All data generated or analyzed during the present study are included in this published article.

\section{Authors' contributions}

$\mathrm{XZ}$ analyzed the mRNA expression and the prognosis data, and contributed to the writing of the manuscript. CRY analyzed the bioinformatics data and constructed the figures and tables. JS and MHZ instructed the whole research study and revised the manuscript. All authors read and approved the final manuscript.

\section{Ethics approval and consent to participate}

No applicable.

\section{Patient consent for publication}

Not applicable.

\section{Competing interests}

The authors declare that they have no competing interests.

\section{References}

1. Siegel RL, Miller KD and Jemal A: Cancer statistics, 2017. CA Cancer J Clin 67: 7-30, 2017.

2. Balakrishnan M, George R, Sharma A and Graham DY: Changing trends in stomach cancer throughout the world. Curr Gastroenterol Rep 19: 36, 2017.

3. Goodenough DA and Paul DL: Gap junctions. Cold Spring Harb Perspect Biol 1: a002576, 2009.

4. Sohl G and Willecke K: Gap junctions and the connexin protein family. Cardiovasc Res 62: 228-232, 2004.

5. Naoi Y, Miyoshi Y, Taguchi T, Kim SJ, Arai T, Tamaki Y and Noguchi S: Connexin26 expression is associated with lymphatic vessel invasion and poor prognosis in human breast cancer. Breast Cancer Res Treat 106: 11-17, 2007.

6. Elzarrad MK, Haroon A, Willecke K, Dobrowolski R, Gillespie MN and Al-Mehdi AB: Connexin-43 upregulation in micrometastases and tumor vasculature and its role in tumor cell attachment to pulmonary endothelium. BMC Med 6: 20, 2008.

7. Laird DW: The gap junction proteome and its relationship to disease. Trends Cell Biol 20: 92-101, 2010.

8. Naus CC and Laird DW: Implications and challenges of connexin connections to cancer. Nat Rev Cancer 10: 435-441, 2010.

9. Czyz J: The stage-specific function of gap junctions during tumourigenesis. Cell Mol Biol Lett 13: 92-102, 2008.

10. Szasz AM, Lánczky A, Nagy A, Förster S, Hark K, Green JE, Boussioutas A, Busuttil R, Szabo A and Gyorffy B: Cross-validation of survival associated biomarkers in gastric cancer using transcriptomic data of 1,065 patients. Oncotarget 7: 49322-49333, 2016.

11. Jiang JX and Gu S: Gap junction- and hemichannel-independent actions of connexins. Biochim Biophys Acta 1711: 208-214, 2005.

12. Beyer EC and Berthoud VM: Gap junction structure: Unraveled, but not fully revealed. F1000Res 6: 568, 2017.

13. Puebla C, Cisterna BA, Salas DP, Delgado-Lopez F, Lampe PD and Saez JC: Linoleic acid permeabilizes gastric epithelial cells by increasing connexin 43 levels in the cell membrane via a GPR40- and Akt-dependent mechanism. Biochim Biophys Acta 1861: 439-448, 2016.

14. Fasciani I, Temperan A, Perez-Atencio LF, Escudero A, Martinez-Montero P, Molano J, Gomez-Hernandez JM, Paino CL, Gonzalez-Nieto D and Barrio LC: Regulation of connexin hemichannel activity by membrane potential and the extracellular calcium in health and disease. Neuropharmacology 75: 479-490, 2013.

15. Maes M, Cogliati B, Crespo Yanguas S, Willebrords J and Vinken M: Roles of connexins and pannexins in digestive homeostasis. Cell Mol Life Sci 72: 2809-2821, 2015.

16. Carette D, Gilleron J, Chevallier D, Segretain D and Pointis G: Connexin a check-point component of cell apoptosis in normal and physiopathological conditions. Biochimie 101: 1-9, 2014.

17. Liu X, Furuya T, Li D, Xu J, Cao X, Li Q, Xu J, Xu Z, Sasaki K and Liu X: Connexin 26 expression correlates with less aggressive phenotype of intestinal type-gastric carcinomas. Int J Mol Med 25: 709-716, 2010.

18. Wang Y, Huang LH, Xu CX, Xiao J, Zhou L, Cao D, Liu XM and Qi Y: Connexin 32 and 43 promoter methylation in Helicobacter pylori-associated gastric tumorigenesis. World J Gastroenterol 20: 11770-11779, 2014.

19. Liu X, Cao K, Xu C, Hu T, Zhou L, Cao D, Xiao J, Luo L, Guo Y and Qi Y: GATA-3 augmentation down-regulates Connexin43 in Helicobacter pylori associated gastric carcinogenesis. Cancer Biol Ther 16: 987-996, 2015.

20. Yahiro K, Hirayama T, Moss J and Noda M: Helicobacter pylori VacA toxin causes cell death by inducing accumulation of cytoplasmic connexin 43. Cell Death Dis 6: e1971, 2015.

21. Radin JN, Gonzalez-Rivera C, Frick-Cheng AE, Sheng J, Gaddy JA, Rubin DH, Algood HM, McClain MS and Cover TL: Role of connexin 43 in Helicobacter pylori VacA-induced cell death. Infect Immu 82: 423-432, 2014.

22. Tang B, Peng ZH, Yu PW, Yu G and Qian F: Expression and significance of $\mathrm{Cx} 43$ and E-cadherin in gastric cancer and metastatic lymph nodes. Med Oncol 28: 502-508, 2011. 
23. Tang B, Peng ZH, Yu PW, Yu G, Qian F, Zeng DZ, Zhao YL, Shi Y, Hao YX and Luo HX: Aberrant expression of Cx43 is associated with the peritoneal metastasis of gastric cancer and Cx43-mediated gap junction enhances gastric cancer cell diapedesis from peritoneal mesothelium. PLoS One 8: e74527, 2013.

24. Liu D, Zhou H, Wu J, Liu W, Li Y, Shi G, Yue X, Sun X, Zhao Y, $\mathrm{Hu} \mathrm{X}$, et al: Infection by $\mathrm{Cx} 43$ adenovirus increased chemotherapy sensitivity in human gastric cancer BGC-823 cells: Not involving in induction of cell apoptosis. Gene 574: 217-224, 2015.

25. Yeh ES, Williams CJ, Williams CB, Bonilla IV Klauber-DeMore N and Phillips SL: Dysregulated connexin 43 in HER2-positive drug resistant breast cancer cells enhances proliferation and migration. Oncotarget 8: 109358-109369, 2017.

26. Teleki I, Szasz AM, Maros ME, Gyorffy B, Kulka J, Meggyeshazi N, Kiszner G, Balla P, Samu A and Krenacs T: Correlations of differentially expressed gap junction connexins $\mathrm{Cx} 26, \mathrm{Cx} 30, \mathrm{Cx} 32, \mathrm{Cx} 43$ and $\mathrm{Cx} 46$ with breast cancer progression and prognosis. PLoS One 9: e112541, 2014.

27. Teleki I, Krenacs T, Szasz MA, Kulka J, Wichmann B, Leo C, Papassotiropoulos B, Riemenschnitter C, Moch H and Varga Z: The potential prognostic value of connexin 26 and 46 expression in neoadjuvant-treated breast cancer. BMC Cancer 13: 50, 2013.

28. Banerjee D, Gakhar G, Madgwick D, Hurt A, Takemoto D and Nguyen TA: A novel role of gap junction connexin 46 protein to protect breast tumors from hypoxia. Int J Cancer 127: 839-848, 2010

29. Yuan L, Guo Y, Yi J, Xiao J, Yuan J, Xiong W, Xu H, Yang Z, Zhang J and Deng H: Identification of a novel GJA3 mutation in congenital nuclear cataract. Optom Vis Sci 92: 337-342, 2015.

30. Morel S, Burnier L, Roatti A, Chassot A, Roth I, Sutter E, Galan K, Pfenniger A, Chanson M and Kwak BR: Unexpected role for the human Cx37 C1019T polymorphism in tumour cell proliferation. Carcinogenesis 31: 1922-1931, 2010.

31. Good ME, Nelson TK, Simon AM and Burt JM: A functional channel is necessary for growth suppression by Cx37. J Cell Sci 124: 2448-2456, 2011

32. Jing Y, Guo S, Zhang X, Sun A, Tao F, Ju H and Qian H: Effects of small interfering RNA interference of connexin 37 on subcutaneous gastric tumours in mice. Mol Med Rep 10: 2955-2960, 2014.

33. Sohl G, Joussen A, Kociok N and Willecke K: Expression of connexin genes in the human retina. BMC Ophthalmol 10: 27, 2010.

34. Shen B, Fang T, Dai M, Jones G and Zhang S: Independent losses of visual perception genes Gja10 and Rbp3 in echolocating bats (Order: Chiroptera). PLoS One 8: e68867, 2013.

35. Alvi MA, Liu X, O'Donovan M, Newton R, Wernisch L, Shannon NB, Shariff K, di Pietro M, Bergman JJ, Ragunath K and Fitzgerald RC: DNA methylation as an adjunct to histopathology to detect prevalent, inconspicuous dysplasia and early-stage neoplasia in Barrett's esophagus. Clin Cancer Res 19: 878-888, 2013
36. Finegold DN, Baty CJ, Knickelbein KZ, Perschke S, Noon SE, Campbell D, Karlsson JM, Huang D, Kimak MA, Lawrence EC, et al: Connexin 47 mutations increase risk for secondary lymphedema following breast cancer treatment. Clin Cancer Res 18: 2382-2390, 2012.

37. Kang KS, Yun JW, Yoon B, Lim YK and Lee YS: Preventive effect of germanium dioxide on the inhibition of gap junctional intercellular communication by TPA. Cancer Lett 166: 147-153, 2001.

38. Yamasaki H, Krutovskikh V, Mesnil M, Tanaka T, Zaidan-Dagli ML and Omori Y: Role of connexin (gap junction) genes in cell growth control and carcinogenesis. C R Acad Sci III 322: 151-159, 1999.

39. Yamasaki H, Omori Y, Zaidan-Dagli ML, Mironov N, Mesnil M and Krutovskikh V: Genetic and epigenetic changes of intercellular communication genes during multistage carcinogenesis. Cancer Detect Prev 23: 273-279, 1999.

40. Ruch RJ, Porter S, Koffler LD, Dwyer-Nield LD and Malkinson AM: Defective gap junctional intercellular communication in lung cancer: Loss of an important mediator of tissue homeostasis and phenotypic regulation. Exp Lung Res 27: 231-243, 2001.

41. Trosko JE: The role of stem cells and gap junctional intercellular communication in carcinogenesis. J Biochem Mol Biol 36: 43-48, 2003.

42. Trosko JE and Ruch RJ: Cell-cell communication in carcinogenesis. Front Biosci 3: d208-d236, 1998.

43. Gravalos $\mathrm{C}$ and Jimeno A: HER2 in gastric cancer: A new prognostic factor and a novel therapeutic target. Ann Oncol 19: $1523-1529,2008$

44. Kanczuga-Koda L, Sulkowski S, Lenczewski A, Koda M, Wincewicz A, Baltaziak M and Sulkowska M: Increased expression of connexins 26 and 43 in lymph node metastases of breast cancer. J Clin Pathol 59: 429-433, 2006.

45. Ezumi K, Yamamoto H, Murata K, Higashiyama M, Damdinsuren B, Nakamura Y, Kyo N, Okami J, Ngan CY, Takemasa I, et al: Aberrant expression of connexin 26 is associated with lung metastasis of colorectal cancer. Clin Cancer Res 14: 677-684, 2008.

This work is licensed under a Creative Commons Attribution-NonCommercial-NoDerivatives 4.0 International (CC BY-NC-ND 4.0) License. 